\title{
A super-Earth orbiting the nearby $M$ dwarf GJ 536
}

\author{
A. Suárez Mascareño ${ }^{1,2}$, J. I. González Hernández ${ }^{1,2}$, R. Rebolo ${ }^{1,2,3}$, N. Astudillo-Defru ${ }^{4}$, X. Bonfils ${ }^{5,6}$, F. Bouchy ${ }^{4}$, \\ X. Delfosse ${ }^{5,6}$, T. Forveille ${ }^{5,6}$, C. Lovis ${ }^{4}$, M. Mayor ${ }^{4}$, F. Murgas ${ }^{5,6}$, F. Pepe ${ }^{4}$, N. C. Santos ${ }^{7,8}$, \\ S. Udry ${ }^{4}$, A. Wünsche ${ }^{7,8}$, and S. Velasco ${ }^{1,2}$
}

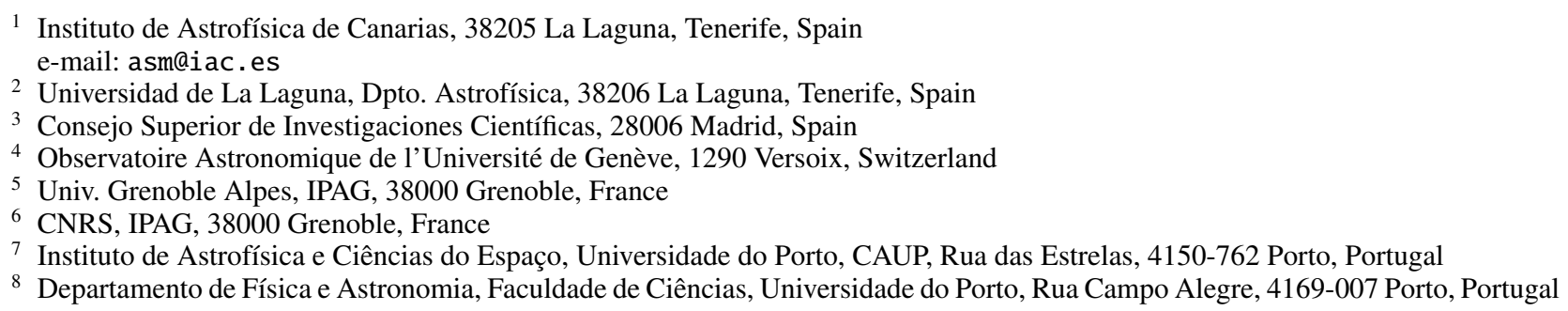

\begin{abstract}
We report the discovery of a super-Earth orbiting the star GJ 536 based on the analysis of the radial-velocity time series from the HARPS and HARPS-N spectrographs. GJ $536 \mathrm{~b}$ is a planet with a minimum mass $M \sin i$ of $5.36 \pm 0.69 M_{\oplus}$; it has an orbital period of $8.7076 \pm 0.0025 \mathrm{~d}$ at a distance of $0.066610(13) \mathrm{AU}$, and an orbit that is consistent with circular. The host star is the moderately quiet M1 V star GJ 536, located at $10 \mathrm{pc}$ from the Sun. We find the presence of a second signal at $43 \mathrm{~d}$ that we relate to stellar rotation after analysing the time series of $\mathrm{Ca}$ II $\mathrm{H} \& \mathrm{~K}$ and $\mathrm{H}_{\alpha}$ spectroscopic indicators and photometric data from the ASAS archive. We find no evidence linking the short period signal to any activity proxy. We also tentatively derived a stellar magnetic cycle of less than 3 yr.
\end{abstract}

Key words. techniques: radial velocities - stars: rotation - planets and satellites: terrestrial planets - stars: individual: GJ 536 stars: activity - planets and satellites: general

\section{Introduction}

Several surveys have attempted to take advantage of the low masses of $\mathrm{M}$ dwarfs and therefore of the stronger radialvelocity signals induced for the same planetary mass - and their closer habitable zones to detect rocky habitable planets (Bonfils et al. 2013; Howard et al. 2014; Irwin et al. 2015; Berta-Thompson et al. 2015a; Anglada-Escudé et al. 2016). While surveying $M$ dwarfs has advantages, it also has its own drawbacks. Stellar activity has been one of the main difficulties when trying to detect planets trough Doppler spectroscopy. Not only does it introduce noise, but also coherent signals that can mimic those of planetary origin (Queloz et al. 2001; Bonfils et al. 2007; Robertson et al. 2014). M dwarfs tend to induce signals with amplitudes comparable to those of rocky planets (Howard et al. 2014; Robertson et al. 2014). While these kinds of stars allow for the detection of smaller planets, they also demand a more detailed analysis of the radial-velocity signals induced by activity. In addition, these low-mass stars offer valuable complementary information on the formation mechanisms of planetary systems. For instance, giants planets are known to be rare around $\mathrm{M}$ dwarfs, while super-Earths appear to

\footnotetext{
* The data used in this paper (Table A.1) are only available at the CDS via anonymous ftp to cdsarc.u-strasbg. fr (130.79.128.5) or via

http://cdsarc.u-strasbg.fr/viz-bin/qcat?J/A+A/597/A108
}

be more frequent (Bonfils et al. 2013; Dressing \& Charbonneau 2013; Dressing et al. 2015).

In spite of the numerous exoplanets detected by Kepler (Howard et al. 2012) and by radial-velocity surveys (Howard et al. 2009; Mayor et al. 2011) the number of known small rocky planets is still comparably low. There are around 1500 confirmed exoplanets and more than 3000 Kepler candidates, but only about a hundred of the confirmed planets have been reported on $\mathrm{M}$ dwarfs and only a fraction of them are rocky planets. The first discovery of a planet around an M dwarf dates back to 1998 (Delfosse et al. 1998; Marcy et al. 1998). Since then several planetary systems have been reported containing Neptune-mass planets and super-Earths (Udry et al. 2007; Delfosse et al. 2013; Howard et al. 2014; Astudillo-Defru et al. 2015) and even some Earth-mass planets (Mayor et al. 2009; Berta-Thompson et al. 2015b; Wright et al. 2016; Affer et al. 2016; Anglada-Escudé et al. 2016). However, the frequency of very low-mass planets around M dwarfs is not well established. In particular, as noted by Bonfils et al. (2013), the frequency of rocky planets at periods shorter than $10 \mathrm{~d}$ is $0.36_{-0.10}^{+0.24}$; it is $0.41_{-0.13}^{+0.54}$ for the habitable zone of the stars. On the other hand Gaidos (2013) estimated that the frequency of habitable rocky planets is $0.46_{-0.15}^{+0.20}$ on a wider spectral sample of Kepler dwarfs, and Kopparapu (2013) gave a frequency of $0.48_{-0.24}^{+0.12}$ for habitable planets around $M$ dwarfs. The three measurements are compatible, but uncertainties are still big making it important to continue the 
Table 1. Stellar parameters of GJ 536.

\begin{tabular}{lll}
\hline \hline Parameter & GJ 536 & Ref. \\
\hline RA $(\mathrm{J} 2000)$ & $14: 01: 03.19$ & 1 \\
Dec $(\mathrm{J} 2000)$ & $-02: 39: 17.52$ & 1 \\
$\delta \mathrm{RA}\left(\mathrm{mas} \mathrm{yr}^{-1}\right)$ & -823.47 & 1 \\
$\delta$ Dec $\left(\mathrm{mas} \mathrm{yr}^{-1}\right)$ & 598.19 & 1 \\
Distance $[\mathrm{pc}]$ & 10.03 & 1 \\
$m_{B}$ & 11.177 & 2 \\
$m_{V}$ & 9.707 & 2 \\
$m_{V}$ ASAS & 9.708 & 0 \\
Spectral type & $\mathrm{M} 1$ & 3 \\
$T_{\text {eff }}[\mathrm{K}]$ & $3685 \pm 68$ & 3 \\
{$[\mathrm{Fe} / \mathrm{H}]$} & $-0.08 \pm 0.09$ & 3 \\
$M_{\star}\left[M_{\odot}\right]$ & $0.52 \pm 0.05$ & 3 \\
$R_{\star}\left[R_{\odot}\right]$ & $0.50 \pm 0.05$ & 3 \\
$\log g(\mathrm{cgs})$ & $4.75 \pm 0.04$ & 3 \\
$\log \left(L_{\star} / L_{\odot}\right)$ & -1.377 & 3 \\
$\log \left(R_{\mathrm{HK}}^{\prime}\right)$ & $-5.12 \pm 0.05$ & 0 \\
$P_{\text {rot }}$ & $45.39 \pm 1.33$ & 0 \\
$v \sin i\left(\mathrm{~km} \mathrm{~s}^{-1}\right)$ & $<1.2^{*}$ & 0 \\
$\operatorname{Secular~acc.~}\left(\mathrm{m} \mathrm{s}^{-1} \mathrm{yr}^{-1}\right)$ & 0.24 & 4 \\
\hline
\end{tabular}

Notes. ${ }^{(*)}$ Estimated using the Radius estimated by Maldonado et al. (2015) and our period determination.

References. 0 - This work; 1 - van Leeuwen (2007); 2 - Koen et al. (2010); 3 - Maldonado et al. (2015); 4 - Calculated following Montet et al. (2014).

search for planets around this star type in order to refine the statistics.

We present the discovery of a super-Earth orbiting the nearby star GJ 536, which is a high proper motion early $M$ dwarf at a distance of $10 \mathrm{pc}$ from the Sun (van Leeuwen 2007; Maldonado et al. 2015). Because of its high proper motion and its closeness, this star shows a secular acceleration of $0.24 \mathrm{~m} \mathrm{~s}^{-1} \mathrm{yr}^{-1}$ (Montet et al. 2014). Table 1 shows the stellar parameters. Its moderately low activity combined with its long rotation period of more than $40 \mathrm{~d}$ (Suárez Mascareño et al. 2015) makes it a very interesting candidate to search for rocky planets.

The star GJ 536 is part of the Bonfils et al. (2013) sample and has been extensively monitored since mid-2004. We have used 146 HARPS spectra taken over $11.7 \mathrm{yr}$ along with 12 HARPS-N spectra taken during April and May 2016. HARPS (Mayor et al. 2003) and HARPS-N (Cosentino et al. 2012) are two fibre-fed high-resolution echelle spectrographs installed at the $3.6 \mathrm{~m} \mathrm{ESO}$ telescope in La Silla Observatory (Chile) and at the Telescopio Nazionale Galileo in the Roque de los Muchachos Observatory (Spain), respectively. Both instruments have a resolving power greater than $R \sim 115000$ over a spectral range from $\sim 380$ to $\sim 690 \mathrm{~nm}$ and have been designed to attain very high longterm radial-velocity accuracy. Both are contained in vacuum vessels to avoid spectral drifts due to temperature and air pressure variations, thus ensuring their stability. HARPS and HARPS-N are equipped with their own pipeline providing extracted and wavelength-calibrated spectra, as well as RV measurements and other data products such as cross-correlation functions (CCFs) and their bisector profiles.

Most of the observations were carried out using the Fabry Pérot interferometer (FP) as simultaneous calibration. The FP offers the possibility of monitoring the instrumental drift with a precision of $10 \mathrm{~cm} \mathrm{~s}^{-1}$ without the risk of contamination of the stellar spectra by the ThAr saturated lines (Wildi et al. 2010). While this is not usually a problem in $\mathrm{G}$ and $\mathrm{K}$ stars, the small amount of light collected in the blue part of the spectra of $\mathrm{M}$ dwarfs might compromise the quality of the measurement of the Ca II H\&K flux. The FP allows a precision of $\sim 1 \mathrm{~m} \mathrm{~s}^{-1}$ in the determination of the radial velocities of the spectra with highest signal-to-noise ratios while assuring the quality of the spectroscopic indicators even in those spectra with low signal-to-noise ratios. Measurements taken before the availability of the FP were taken without simultaneous reference.

We also use the photometric data on GJ 536 provided by the All Sky Automated Survey (ASAS) public database. ASAS (Pojmanski 1997) is an all-sky survey in the $V$ and $I$ bands running since 1998 at Las Campanas Observatory, Chile. The best photometric results are achieved for stars with $V \sim 8-14$, but this range can be extended by implementing some quality control on the data. ASAS has produced light curves for around $10^{7}$ stars at $\delta<28^{\circ}$. The ASAS catalogue supplies ready-to-use light curves with flags indicating the quality of the data. For this analysis we relied only on good quality data (grade A and B in the internal flags). Even after this quality control, there are still some high dispersion measurements which cannot be explained by a regular stellar behaviour. We reject those measurements by de-trending the series and eliminating points deviating more than three times the standard deviation from the median seasonal value. We are left with 359 photometric observations taken over $8.6 \mathrm{yr}$ with a typical uncertainty of 9.6 mmag per exposure.

\section{Determining stellar activity indicators and radial velocities}

\subsection{Activity indicators}

For the activity analysis we use the extracted order-by-order wavelength-calibrated spectra produced by the HARPS and HARPS-N pipelines. For a given star, the change in atmospheric transparency from day to day causes variations in the flux distribution of the recorded spectra that are particularly relevant in the blue where we intend to measure Ca II lines. In order to minimize the effects related to these atmospheric changes we create a spectral template for each star by de-blazing and coadding every available spectrum; we use the co-added spectrum to correct the order-by-order flux of each individual spectrum. We also correct each spectrum for the Earth's barycentric radial velocity and the radial velocity of the star using the measurements given by the standard pipeline, and re-binned the spectra into a wavelength-constant step. Using this HARPS dataset, we expect to have high-quality spectroscopic indicators to monitor tiny stellar activity variations with high accuracy.

\section{$S_{\text {MW }}$ index}

We calculate the Mount Wilson $S$ index and the $\log _{10}\left(R_{H K}^{\prime}\right)$ by using the original Noyes et al. (1984) procedure, following Lovis et al. (2011) and Suárez Mascareño et al. (2015). We define two triangular passbands with full width half maximum (FWHM) of $1.09 \AA$ centred at $3968.470 \AA$ and $3933.664 \AA$ for the Ca II H\&K line cores, and for the continuum we use two $20 \AA$ wide bands centred at 3901.070 $\AA$ (V) and 4001.070 $\AA(\mathrm{R})$, as shown in Fig. 1.

Then the $S$-index is defined as

$S=\alpha \frac{\tilde{N}_{H}+\tilde{N}_{K}}{\tilde{N}_{R}+\tilde{N}_{V}}+\beta$ 

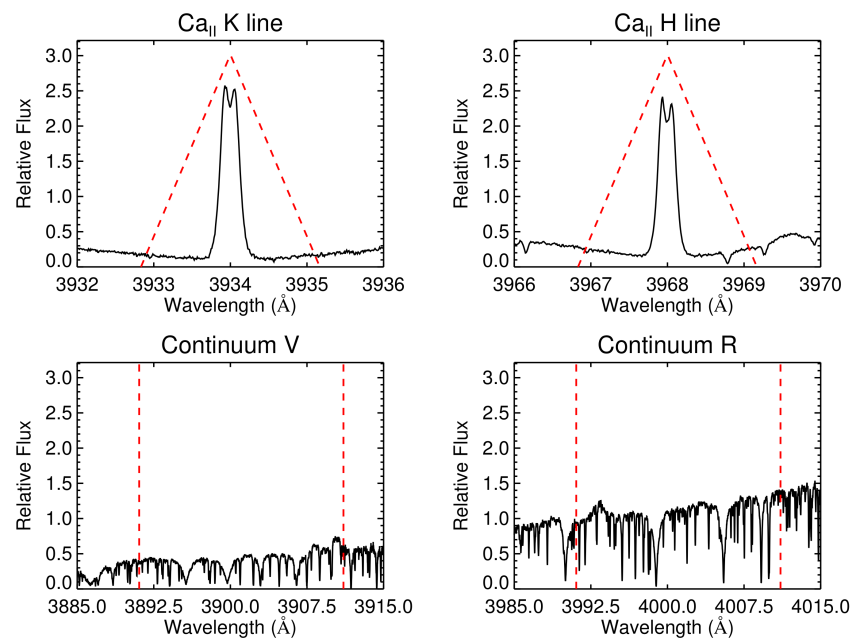

Fig. 1. Ca II H\&K filter of the spectrum of the star GJ536 with the same shape as the Mount Wilson Ca II H\&K passband.

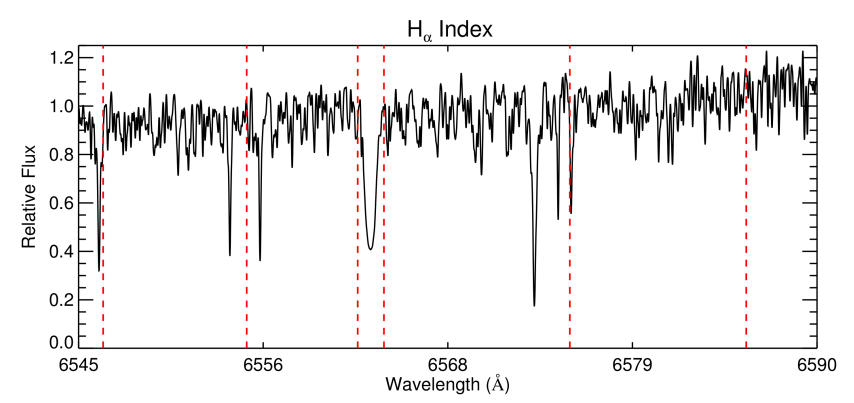

Fig. 2. Spectrum of the M-type star GJ536 showing the $\mathrm{H} \alpha$ filter passband and continuum bands.

where $\tilde{N}_{H}, \tilde{N}_{K}, \tilde{N}_{R}$, and $\tilde{N}_{V}$ are the mean fluxes in each passband, while $\alpha$ and $\beta$ are calibration constants fixed as $\alpha=1.111$ and $\beta=0.0153$. The $S$ index serves as a measurement of the $\mathrm{Ca}$ II H\&K core flux normalized to the neighbour continuum. As a normalized index to compare it to other stars, we compute the $\log _{10}\left(R_{\mathrm{HK}}^{\prime}\right)$ following Suárez Mascareño et al. (2015).

\section{$H_{\alpha}$ index}

We also use the $\mathrm{H} \alpha$ index, with a simpler passband following Gomes da Silva et al. (2011). It consists of a rectangular bandpass with a width of $1.6 \AA$ and centred at $6562.808 \AA$ (core), and two continuum bands of $10.75 \AA$ and $8.75 \AA$ in width centred at $6550.87 \AA(\mathrm{L})$ and $6580.31 \AA(\mathrm{R})$, respectively, as seen in Fig. 2 .

Thus, the $\mathrm{H} \alpha$ index is defined as

$H \alpha_{\text {Index }}=\frac{H \alpha_{\text {core }}}{H \alpha_{L}+H \alpha_{R}}$.

\subsection{Radial velocities}

The radial-velocity measurements in the HARPS standard pipeline is determined by a Gaussian fit of the CCF of the spectrum with a binary stellar template (Baranne et al. 1996; Pepe et al. 2000). In the case of $M$ dwarfs, owing to the huge number of line blends, the CCF is not Gaussian and results in a less precise Gaussian fit which might cause distortions in the radial-velocity measurements and FWHM. To deal with this issue we tried two different approaches.

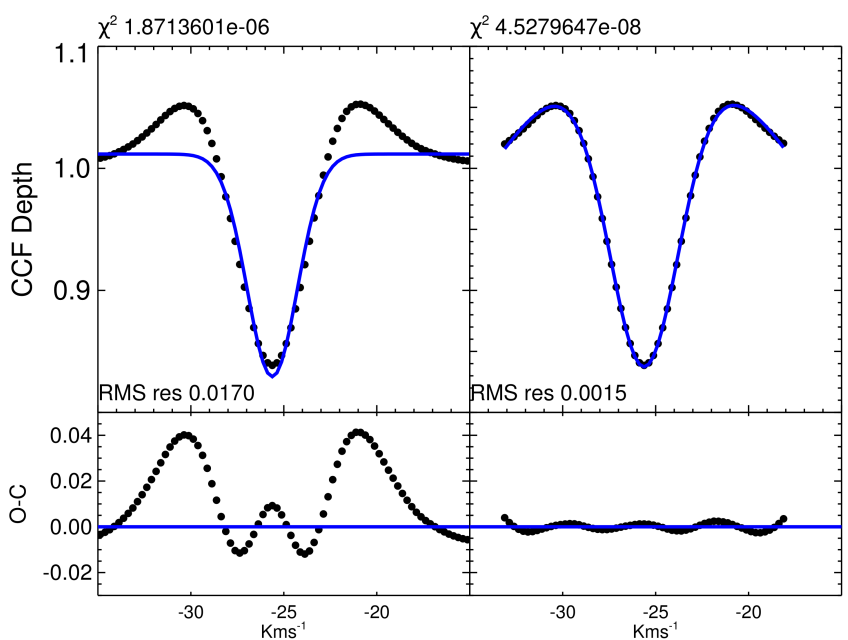

Fig. 3. Cross-correlation function for GJ 536. Upper panels show the CCF with the Gaussian fit (left) and our Gaussian plus polynomial fit (right). Lower panels show the residuals after the fit for the Gaussian fit (left) and for our fit. Blue lines show the fit (upper panels) and the zero line (lower panels).

The first approach consisted in using a slightly more complex model for the CCF fitting, a Gaussian function plus a secondorder polynomial (Fig. 3) using only the central region of the $\mathrm{CCF}$ function. We use a $15 \mathrm{~km} \mathrm{~s}^{-1}$ window centred at the minimum of the CCF. This configuration provides the best stability of the measurements. Along with the measurements of the radial velocity we obtain the FWHM of the cross-correlation function, which we also use to track variations in the activity level of the star. The second approach to the problem was to recompute the radial velocities using a template matching algorithm with a high signal-to-noise stellar spectral template (Astudillo-Defru et al. 2015). Every spectrum is corrected from both barycentric and stellar radial velocity to align it to the frame of the solar system barycentre. The radial velocities are computed by minimizing the $\chi^{2}$ of the residuals between the observed spectra and shifted versions of the stellar template, with all the elements contaminated by telluric lines masked. All radial-velocity measurements are corrected from the secular acceleration of the star.

For the bisector span measurement we rely on the pipeline results as it does not depend on the fit, but on the CCF itself. The bisector has been a standard activity diagnostic tool for solartype stars for more than $10 \mathrm{yr}$. Unfortunately, its behaviour in slow rotating stars is not as informative as it is for fast rotators (Saar \& Donahue 1997; Bonfils et al. 2007). We report the measurements of the bisector span (BIS) for each radial-velocity measurement, but we do not find any meaningful information in its analysis.

\subsection{Quality control of the data}

As the sampling rate of our data is not well suited for modelling fast events, such as flares, and their effect on the radial velocity is not well understood, we identify and reject points likely affected by flares by searching for abnormal behaviour of the activity indicators (Reiners 2009). The process rejected six spectra that correspond to flare events of the star with obvious activity enhancement and line distortion. That leaves us with 140 HARPS spectroscopic observations taken over $10.7 \mathrm{yr}$, with most of the measurements taking place after 2013, with a typical exposure of $900 \mathrm{~s}$ and an average signal-to-noise ratio of 56 at $5500 \AA$. 


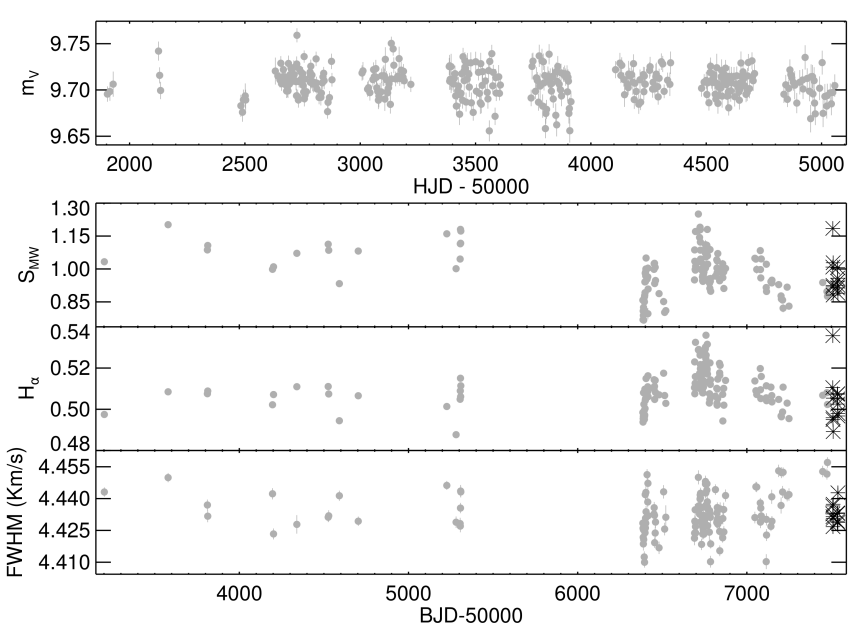

Fig. 4. From top to bottom: time series of the $m_{V}, S_{M W}$ index, $\mathrm{H}_{\alpha}$ index, and FWHM time series. Grey dots show HARPS-S data; black asterisks show HARPS-N data.

We do not apply the quality control procedure to the HARPS-N data as the number of spectra is not high enough.

\section{Stellar activity analysis}

In order to properly understand the behaviour of the star, our first step is to analyse the different modulations present in the photometric and spectroscopic time-series.

We search for periodic variability compatible with both stellar rotation and long-term magnetic cycles. We compute the power spectrum using a generalised Lomb-Scargle periodogram (Zechmeister \& Kürster 2009) and if there is any significant periodicity we fit the detected period using a sinusoidal model, or a double sinusoidal model to account for the asymmetry of some signals (Berdyugina \& Järvinen 2005) with the MPFIT routine (Markwardt 2009).

The significance of the periodogram peak is evaluated using the Cumming (2004) modification of the Horne \& Baliunas (1986) formula to obtain the spectral density thresholds for the desired false alarm probability (FAP) levels and the bootstrap randomization (Endl et al. 2001) of the data.

Figure 4 shows the time series for the photometry (top panel) and the three activity proxies (bottom panels) used for this analysis. The periodograms of both the photometric and FWHM time series show significant signals at $\sim 40 \mathrm{~d}$, compatible with the typical rotation periods of low-activity M1 stars (Suárez Mascareño et al. 2016; Newton et al. 2016). On the other hand, the periodograms of the $S_{M W}$ and $\mathrm{H}_{\alpha}$ indexes show long-term and short-term significant signals. The short period signal is again at $\sim 40 \mathrm{~d}$, while the long-term signal is close to $\sim 1000 \mathrm{~d}$.

\subsection{Long-term magnetic cycle}

Analysing the $S_{M W}$ and $\mathrm{H}_{\alpha}$ indexes time series we find the presence of a long-term magnetic cycle of $\sim 3$ yr. Figure 5 shows the periodograms of the time series of both indexes. We see a well-defined peak in the $S_{M W}$ index periodogram at $\sim 806 \mathrm{~d}$ and several peaks going from $\sim 600 \mathrm{~d}$ to $1100 \mathrm{~d}$ in the $\mathrm{H}_{\alpha}$ index periodogram implying that the shape of the cycle is still not well defined within our observations. Table 2 shows the periods of the best fits for both time series using least-squares minimization with the period corresponding to the highest peak of

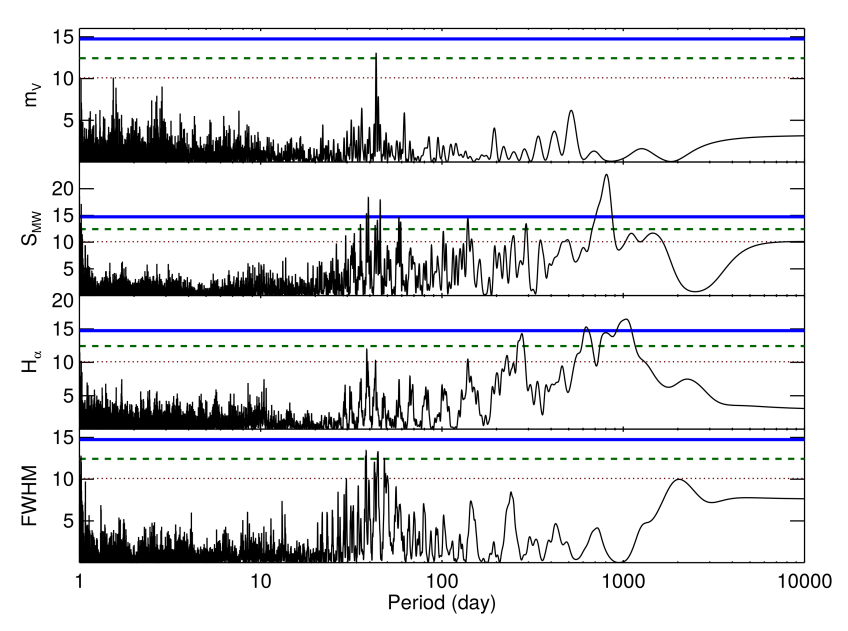

Fig. 5. From top to bottom: periodograms of the $m_{V}, S_{M W}$ index, $\mathrm{H}_{\alpha}$ index, and FWHM time series. Horizontal lines show the different levels of false alarm probability. Red dotted line for a $10 \%$ false alarm probability, green dashed line for $1 \%$, and blue thick line for $0.1 \%$. Several peaks arise with significances better than $0.1 \%$.

the periodogram as the initial guess. Figure 6 shows the phase folded curves using these periods. The two estimates differ significantly. This might be because of a sub-optimal sampling to detect signals of long periods. The detected periodicities might not be the true periodicities, but apparent periodicities close to the real one caused by the sampling. This also makes us think that the uncertainties in the cycle length are underestimated. The length of the signal is shorter than the typical magnetic cycles measured in solar-type stars, but is within the range of known magnetic cycles in M-type stars (Suárez Mascareño et al. 2016). In the $S_{M W}$ and the $\mathrm{H}_{\alpha}$ indexes it seems that the cycle shape shows a quick rise followed by a slow decline, as is the case in the Sun and many other main sequence stars (Waldmeier 1961; Baliunas et al. 1995). Unfortunately this cycle is not well covered in phase, making it difficult to properly characterise it. More observations are needed in order to better constrain its period.

\subsection{Rotation}

The other activity signal expected in our data is the rotational modulation of the star. It shows up at $\sim 43 \mathrm{~d}$ with a false alarm probability close to or smaller than $1 \%$ in the four time series (Fig. 5) that grow in significance after removing the long-term effects.

In the photometric light curve we measure a modulation of $43.33 \pm 0.06 \mathrm{~d}$ with an amplitude of $5.21 \pm 0.68 \mathrm{mmag}$. For the $S_{M W}$ index we find a signal of $43.84 \pm 0.01 \mathrm{~d}$ with an amplitude of $0.0628 \pm 0.0010$ when doing a simultaneous fit with the 824-d signal from Table 6 . In the case of the $\mathrm{H}_{\alpha}$ index we find a signal $42.58 \pm 0.08 \mathrm{~d}$ with an amplitude of $0.0042 \pm 0.0010$, also when doing a simultaneous fit with the $\sim 1075 \mathrm{~d}$ signal. The time series of the FWHM show a linear increase with time of $\sim 2 \mathrm{~m} \mathrm{~s}^{-1} \mathrm{yr}^{-1}$, which might be related to a slow focus drift of HARPS. After subtracting the linear trend we again find a periodicity of $44.47 \pm$ $0.03 \mathrm{~d}$ period with an amplitude of $4.56 \pm 0.31 \mathrm{~m} \mathrm{~s}^{-1}$. Figure 7 shows the phase folded fits of all the signals. The $S_{M W}$ index and FWHM signals seem to be in phase, while the photometric signal is shifted by a quarter phase. The uncertainty in the $\mathrm{H}_{\alpha}$ long-term fit makes it difficult to give it a unique phase to the rotation signal. Table 2 shows the parameters for the four signals. 

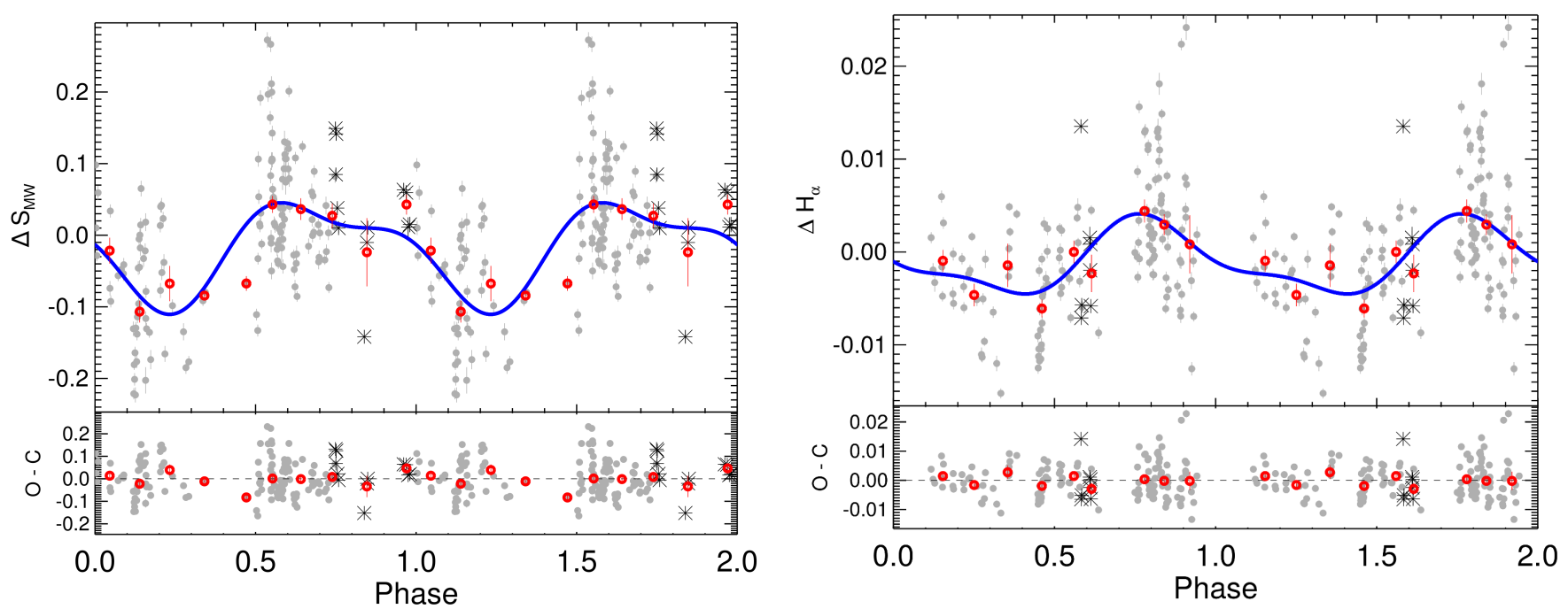

Fig. 6. Phase folded fit for the isolated long period activity signal using double-harmonic sine curves. The left panel shows the $S_{M W}$ index data using the $824 \mathrm{~d}$ signal, while the right panel shows the $\mathrm{H}_{\alpha}$ signal using the $1075 \mathrm{~d}$ signal. Grey dots are the raw measurements after subtracting the mean value. Red dots are the same points binned in phase with a bin size of 0.1 .

Table 2. Magnetic cycle and rotation periodicities.

\begin{tabular}{llll}
\hline \hline Series & Period (d) & Amplitude & FAP (\%) \\
\hline$S_{M W \text { Cyc }}$ & $824.9 \pm 1.7$ & $0.0684 \pm 0.0011$ & $<0.1$ \\
$\mathrm{H}_{\alpha \text { Cyc }}$ & $1075.8 \pm 36.1$ & $0.0046 \pm 0.0011$ & $<0.1$ \\
& & & \\
$m_{V \text { Rot }}$ & $43.33 \pm 0.06$ & $5.21 \pm 0.68 \mathrm{mmag}$ & $<1$ \\
$S_{M W \text { Rot }}$ & $43.84 \pm 0.01$ & $0.0628 \pm 0.0010$ & $<0.1$ \\
$\mathrm{H}_{\alpha \text { Rot }}$ & $42.58 \pm 0.08$ & $0.0042 \pm 0.0010$ & $<0.1$ \\
$F W H M_{\text {Rot }}$ & $44.47 \pm 0.03$ & $4.56 \pm 0.31 \mathrm{~m} \mathrm{~s}^{-1}$ & $<1$ \\
& & & \\
$\langle$ Rot. $\rangle$ & $\mathbf{4 3 . 8 7} \pm \mathbf{0 . 8 0}$ & & \\
\hline
\end{tabular}

Notes. The mean value is the weighted mean of all the individual measurements. The error of the mean is the standard deviation of the individual measurements divided by the square root of the number of measurements.

Our measurement of $45.39 \mathrm{~d}$ strengthens the previous estimation of Suárez Mascareño et al. (2015). Having such a clear detection of the rotational modulation in that many indicators over so many years supports the idea that activity regions in at least some M-type stars are stable over long time spans (Robertson et al. 2015).

\section{Radial-velocity analysis}

Our 152 radial-velocity measurements have a median error of $1.33 \mathrm{~m} \mathrm{~s}^{-1}$ which includes both photon noise, calibration, and telescope related errors. We measure a total systematic radial velocity of $-25.622 \mathrm{~km} \mathrm{~s}^{-1}$ with a dispersion of $3.28 \mathrm{~m} \mathrm{~s}^{-1}$. Figure 8 shows the measured radial velocities. An F-test (Zechmeister et al. 2009) returns a negligible probability (smaller than the $0.1 \%$ ) that the internal errors explain the measured dispersion.

To search for periodic radial-velocity signals in our timeseries we follow a similar procedure to the one explained in Sect. 3.1. We search for periodic signals using a generalised Lomb-Scargle periodogram, and if there is any significant periodicity we fit the detected signal using the RVLIN package (Wright \& Howard 2012). We sequentially find the dominant components in the time series and remove them until no significant signal remains.

Following this procedure we identify one signal with a false alarm probability much higher than $0.1 \%$, using both the bootstrap and the Cumming (2004) estimates, corresponding to a period of $8.7 \mathrm{~d}$ with a semi-amplitude of $2.47 \mathrm{~m} \mathrm{~s}^{-1}$ consistent with circular (Fig. 9 shows the periodogram). Removing this signal leaves a $43.9 \mathrm{~d}$ signal with a semi-amplitude of $2.86 \mathrm{~m} \mathrm{~s}^{-1}$ and an eccentricity of 0.57 , with a false alarm probability better than $0.1 \%$. No further significant signals are found after removing these two (Fig. 9). Figure 10 shows the phase folded fits of both the $8.7 \mathrm{~d}$ and the $43.9 \mathrm{~d}$ signals.

We tested the available dataset for the three ways of calculating the radial velocity, obtaining virtually the same results in every case. Results are shown for the Gaussian + polynomial fit of the cross-correlation function.

\subsection{Origin of the periodic radial-velocity signals}

Stellar activity can induce radial-velocity signals similar to those of Keplerian origin. The inhomogeneities in the surface of the star cause radial-velocity shifts due to the distortion of the spectral line shapes which can, in some cases, create a radial-velocity signal with a periodicity close to the stellar rotation and its first harmonic.

For this star we have a rotation period of $45.39 \pm 1.33 \mathrm{~d}$, and two radial-velocity signals of $8.7 \mathrm{~d}$ and $43.9 \mathrm{~d}$. The second signal matches almost perfectly the rotation period of the star. On the other hand we do not see in the time series of activity indicators any signal close to the $8.7 \mathrm{~d}$. This is the first evidence of the stellar origin of the $43.9 \mathrm{~d}$ signal, and the planetary origin of the $8.7 \mathrm{~d}$ signal.

As a second test we measured the Spearman correlation coefficient between the $S_{M W}$, the $\mathrm{H}_{\alpha}$ index, the FWHM, and the radial velocities. We find a significant correlation between all the indexes and the raw radial velocity, which almost disappears when we isolate the $8.7 \mathrm{~d}$ signal, and slightly increases when isolating the $43.9 \mathrm{~d}$ signal (see Table 3 ). This constitutes a second 

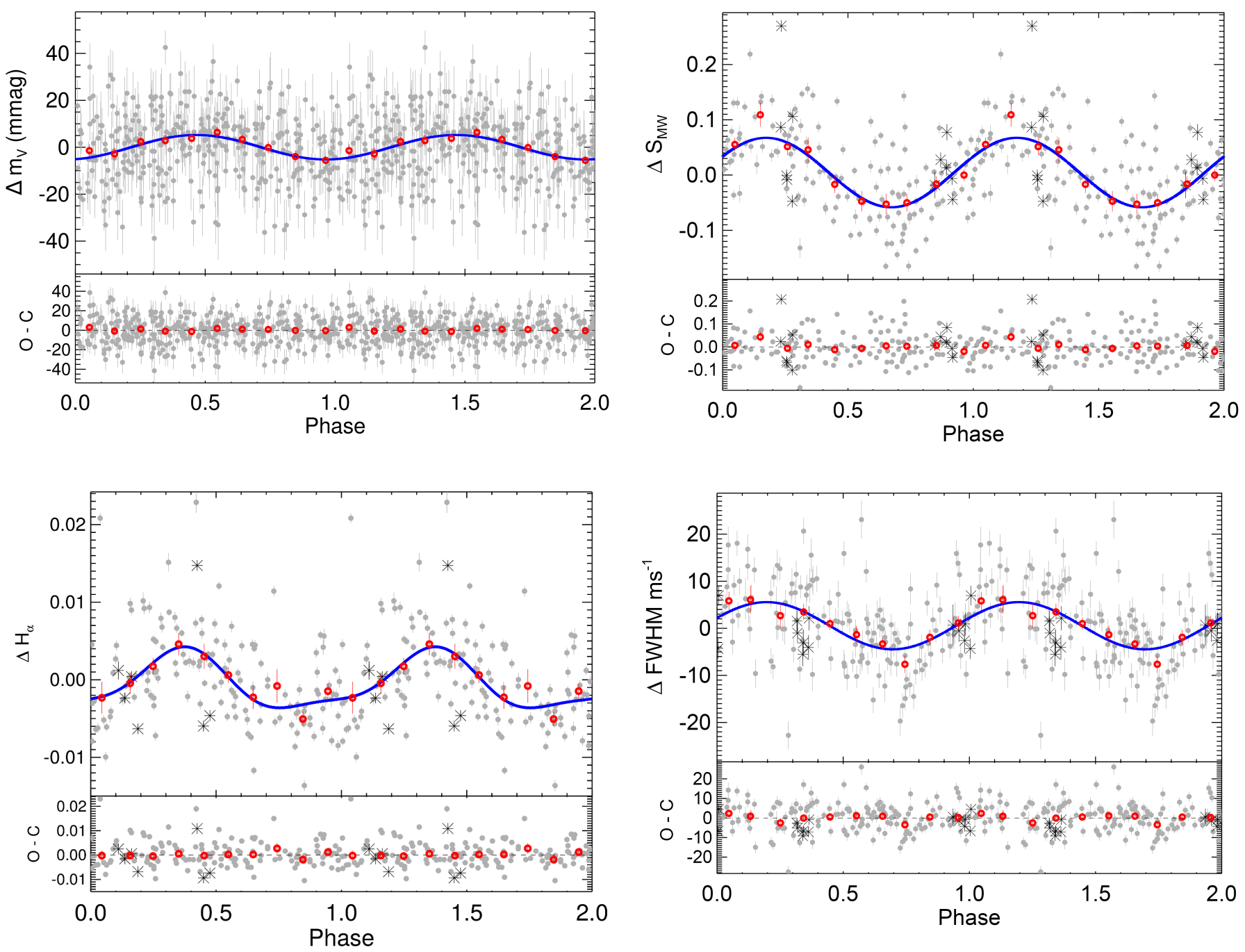

Fig. 7. Phase folded curve using the rotational modulation for the ASAS light curve (upper left), $S_{M W}$ index (upper right), $\mathrm{H}_{\alpha}$ index using a double-harmonic sine curve (lower left), and FWHM (lower right). Grey dots are the raw measurements after subtracting the mean value. Red dots are the same points binned in phase with a bin size of 0.1. The error bar of a given bin is estimated using the weighted standard deviation of binned measurements divided by the square root of the number of measurements included in this bin. This estimation of the bin error bars assumes white noise, which is justified by the binning in phase, which regroups points that are uncorrelated in time.

Table 3. Activity - radial-velocity correlations.

\begin{tabular}{llll}
\hline \hline Parameter & Raw data & 8.7 d signal & 43.9 d signal \\
\hline$S_{M W}$ vs. $V_{R}$ & $0.292(>3 \sigma)$ & $0.069(<1 \sigma)$ & $0.345(>3 \sigma)$ \\
$\mathrm{H}_{\alpha}$ vs. $V_{R}$ & $0.338(>3 \sigma)$ & $0.113(1 \sigma)$ & $0.321(>3 \sigma)$ \\
FWHM vs. $V_{R}$ & $0.356(>3 \sigma)$ & $0.164(1 \sigma)$ & $0.340(>3 \sigma)$ \\
\hline
\end{tabular}

Notes. Long-term variations of activity indicators have been subtracted. The parenthesis value indicates the significance of the correlation given by the bootstrapping process.

piece of evidence of the stellar origin of the $43.9 \mathrm{~d}$ signal, and of the planetary origin of the $8.7 \mathrm{~d}$ signal. Following this idea, we subtract the linear correlation between the radial velocity and each of the three activity diagnostic indexes. By doing this we see that the strength of the $8.7 \mathrm{~d}$ signal remains constant, or even increases, while the significance of the $43.9 \mathrm{~d}$ is reduced in all cases (see Fig. 11), even getting buried in the noise after correcting for the correlation with the $\mathrm{H}_{\alpha}$ index.
Keplerian signals are deterministic and consistent in time. When measuring one signal, we expect to find that the significance of the detection increases steadily with the number of observations, and that the measured period is stable over time. However, in the case of an activity related signal this is not necessarily the case. As the stellar surface is not static, and the configuration of active regions may change in time, changes in the phase of the modulation and in the detected period are expected. Even the disappearance of the signal at certain seasons is possible. Figure 12 shows the evolution of the false alarm probability of the detection of both isolated signals, as well as the measurement of the most prominent period when isolating them. The $8.7 \mathrm{~d}$ signal increases steadily with time, and once it becomes the most significant signal it never moves again. On the other hand, the behaviour of the $43.9 \mathrm{~d}$ is more erratic, losing significance during the last observations.

Of the two significant radial-velocity signals detected in our data it seems clear that the one at $8.7 \mathrm{~d}$ has a planetary origin, while the one at 43.9 has a stellar activity origin.

The shape of the activity induced radial-velocity signal present in our data is evidently not sinusoidal. A double 


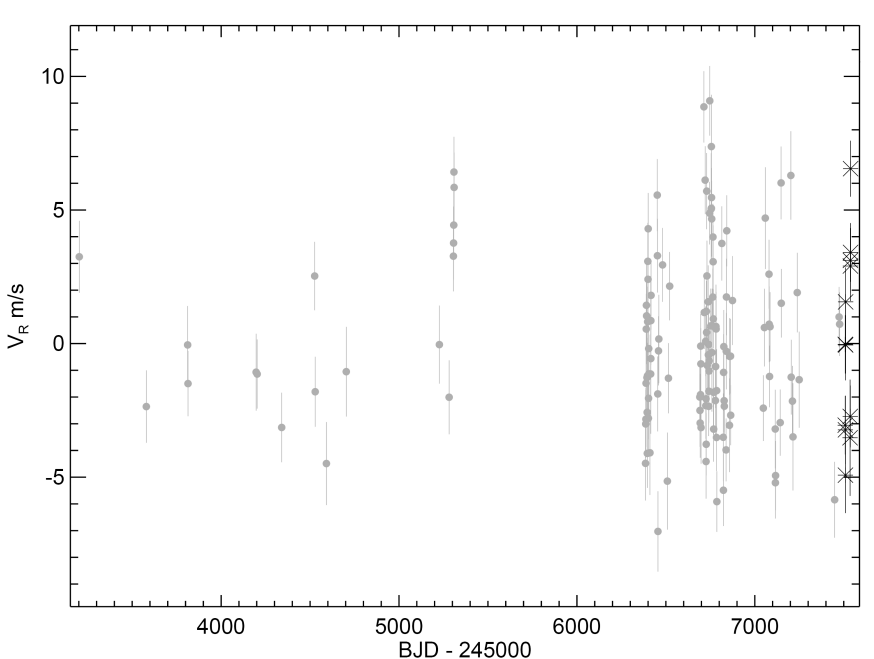

Fig. 8. Radial-velocity time series. Grey dots show HARPS-S data; black asterisks show HARPS-N data.

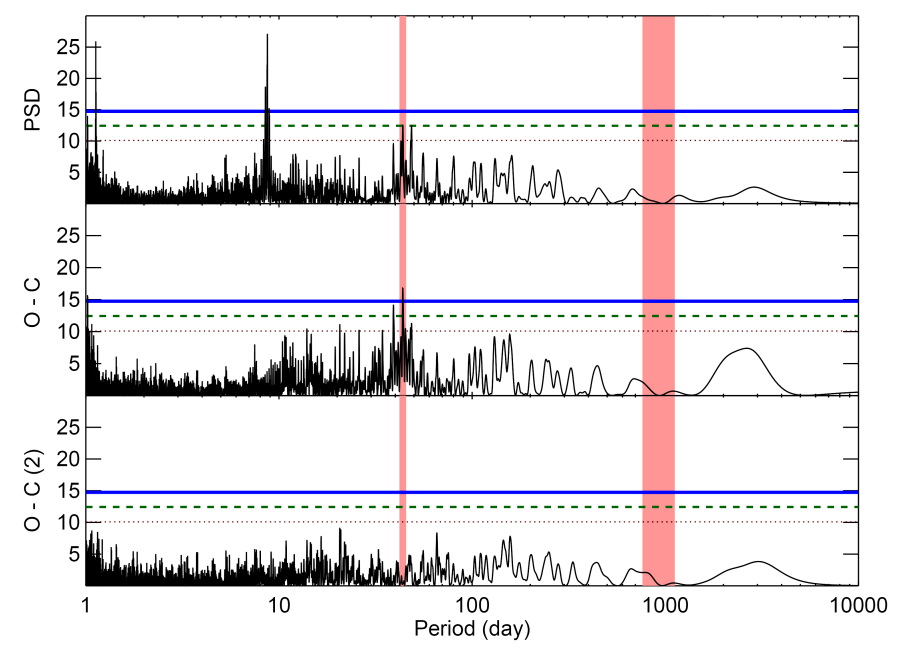

Fig. 9. Periodograms of the radial velocity. The upper panel shows the raw periodogram, the middle panel the periodogram of the residuals after subtracting the $8.7 \mathrm{~d}$ signal, and the lower panel the periodogram of the residuals after subtracting the $43 \mathrm{~d}$ signal present in the middle panel. Red regions show the periods of the measured rotation and magnetic cycle. Red dotted line for a $10 \%$ false alarm probability; green dashed line for $1 \%$, and blue thick line for $0.1 \%$.

harmonic sinusoidal, as in the case of the activity signals, is the best fit model and the only one that does not create ghost signals after subtracting it. The rotation induced signal is not in phase with the rotation signals in the activity indicators. It appears to be shifted by $\sim 45^{\circ}$ from the signal in the $S_{M W}$ index and FWHM time series as seen in Bonfils et al. (2007) and Santos et al. (2014). The uncertainty in the phase $\mathrm{H}_{\alpha}$ time series makes it difficult to measure a reliable phase difference.

Finally, an analysis of the spectral window rules out that the peaks in the periodogram are artefacts of the time sampling alone. No features appear at 8.7 or 43.9 d even after masking the oversaturated regions of the power spectrum. Following Rajpaul et al. (2016) we tried to re-create the 8.7-d by injecting the $P_{\text {Rot }}$ signal along with a second signal at $P_{\text {Rot }} / 2$ at 1000 randomized phase shifts with a white noise model. We were never able to generate a signal at $8.7 \mathrm{~d}$, or any significant signal at periods close to $8.7 \mathrm{~d}$. It seems very unlikely that any of the signals are artefacts of the sampling.
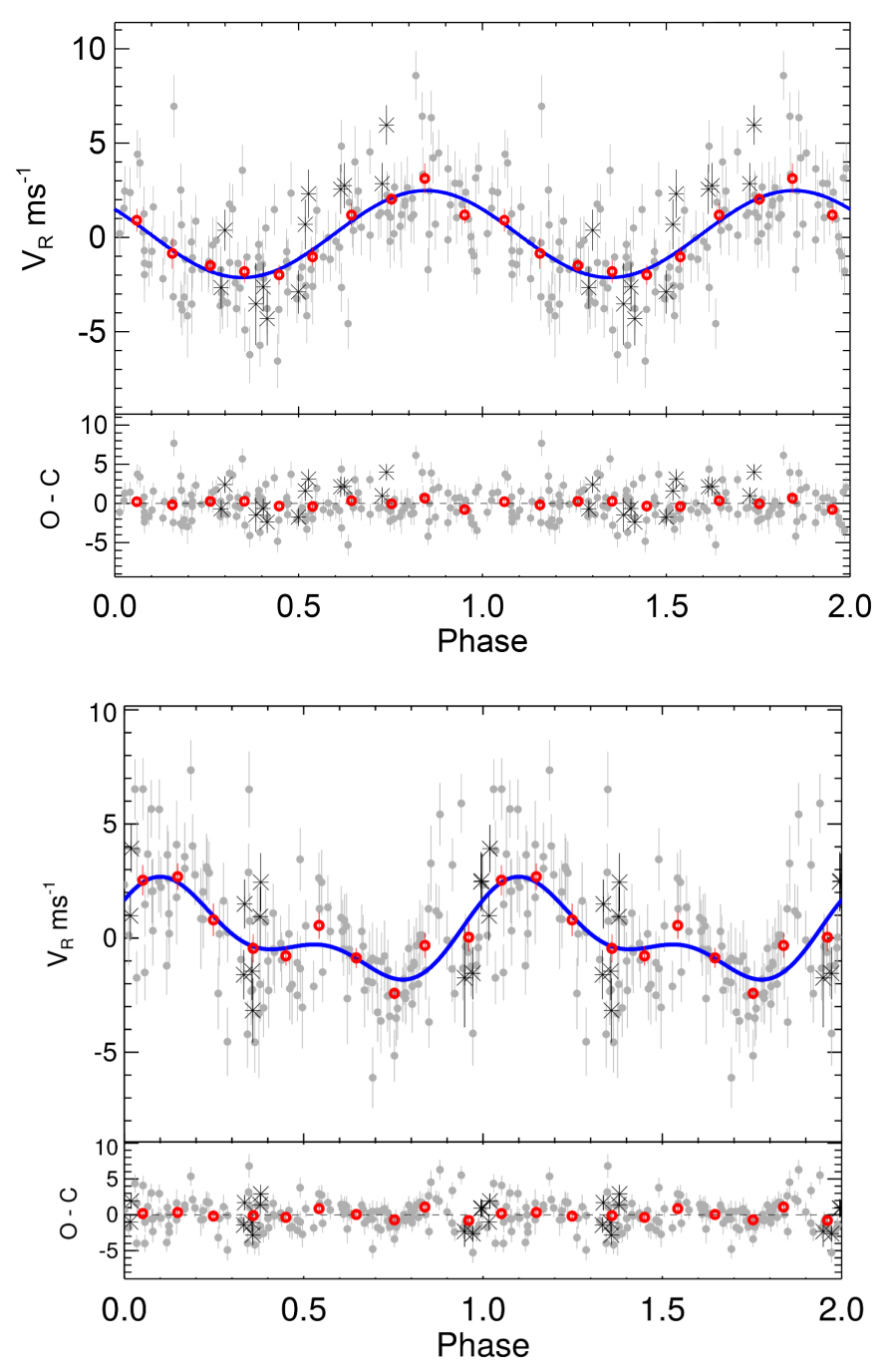

Fig. 10. Top panel: phase folded curve of the radial velocity using the $8.7 \mathrm{~d}$ period. Grey dots are the raw radial-velocity measurements after subtracting the mean value and the $43.9 \mathrm{~d}$ signal. Bottom panel: phase folded curve of the radial velocity using the $43.9 \mathrm{~d}$ period using a double-harmonic sine curve. Grey dots and black asterisks are the raw radial-velocity measurements after subtracting the mean value and the $8.7 \mathrm{~d}$ signal. Red dots are the same points binned in phase with a bin size of 0.1 . The error bar of a given bin is estimated using the weighted standard deviation of binned measurements divided by the square root of the number of measurements included in this bin. This estimation of the bin error bars assumes white noise, which is justified by the binning in phase and which regroups points that are uncorrelated in time.

\subsection{GJ536 b}

The analysis of the radial-velocity time series and of the activity indicators leads us to conclude that the best explanation of the observed data is the existence of a planet orbiting the star GJ 536 with a period of $8.7 \mathrm{~d}$, with a semi-amplitude of $\sim 2.5 \mathrm{~m} \mathrm{~s}^{-1}$. The best solution comes from a super-Earth with a minimum mass of $5.3 M_{\oplus}$ orbiting at $0.067 \mathrm{AU}$ of its star.

\section{MCMC analysis of the radial-velocity time series}

In order to quantify the uncertainties of the orbital parameters of the planet, we perform a Bayesian analysis using the code ExoFIT (Balan \& Lahav 2009). This code follows the Bayesian method described in Gregory (2005), Ford (2005) and 


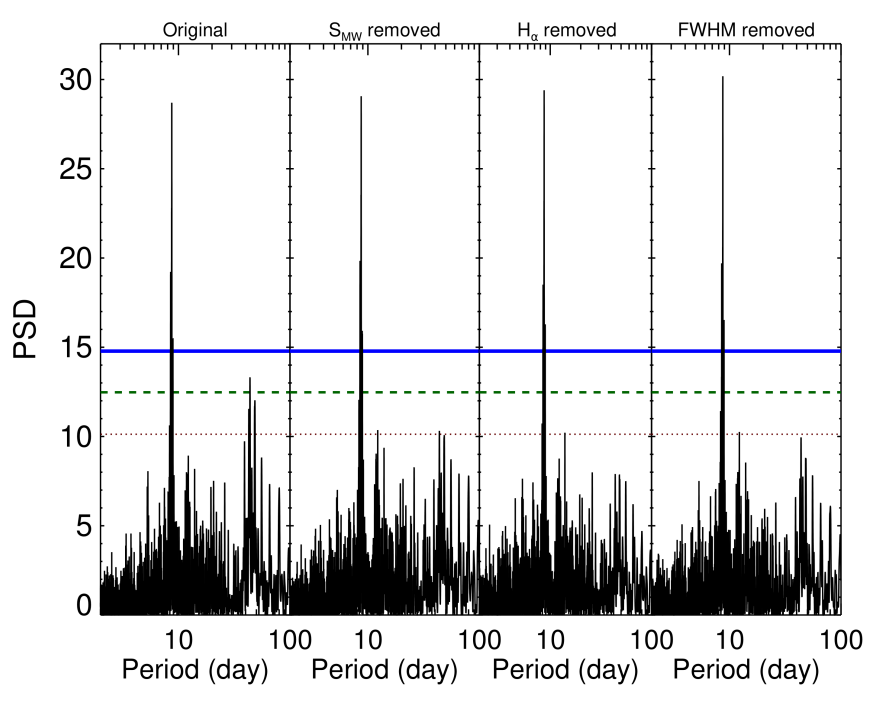

Fig. 11. Periodograms for the radial velocity after removing the correlation with the different activity diagnostic tools. From left to right there is the periodogram for the original data, the periodogram after detrending against the $S_{M W}$ index, against the $\mathrm{H}_{\alpha}$ index, and against the FWHM.
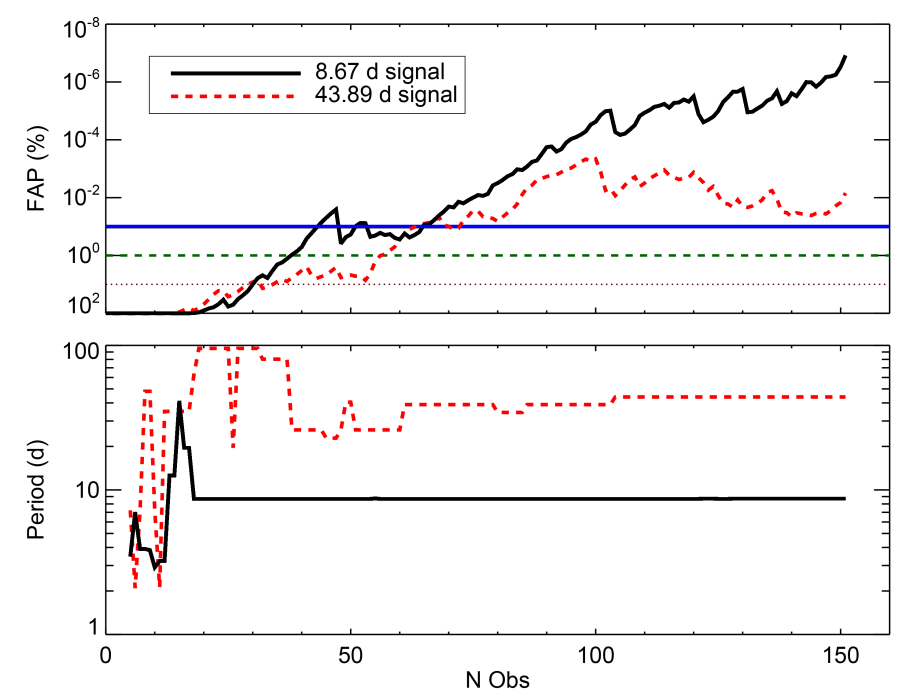

Fig. 12. Evolution of the false alarm probability of the detections (upper panel) for the isolated signals, and stability of the detections (lower panel). Blue thick line shows the behaviour for the $8.7 \mathrm{~d}$ signals and red dashed line for the $43.9 \mathrm{~d}$ signal.

Ford \& Gregory (2007). A single planet can be modelled using the following formula:

$v_{i}=\gamma-K\left[\sin \left(\theta\left(t_{i}+\chi P\right)+\omega\right)+e \sin \omega\right]$,

where $\gamma$ is system radial velocity; $K$ is the velocity semiamplitude equal to $2 \pi P^{-1}\left(1-\mathrm{e}^{2}\right)^{-1 / 2} a \sin i ; P$ is the orbital period; $a$ is the semi-major axis of the orbit; $e$ is the orbital eccentricity; $i$ is the inclination of the orbit; $\omega$ is the longitude of periastron; $\chi$ is the fraction of an orbit, prior to the start of data taking, at which periastron occurs (thus, $\chi P$ equals the number of days prior to $t_{i}=0$ that the star was at periastron, for an orbital period of $P$ days); and $\theta\left(t_{i}+\chi P\right)$ is the angle of the star in its orbit relative to periastron at time $t_{i}$, also called the true anomaly.

To fit the previous equation to the data we need to specify the six model parameters, $P, K, \gamma, e, \omega$, and $\chi$. Observed radialvelocity data, $d_{i}$, can be modelled by the equation $d_{i}=v_{i}+\epsilon_{i}+\delta$
(Gregory 2005), where $v_{i}$ is the modelled radial velocity of the star and $\epsilon_{i}$ is the uncertainty component arising from accountable but unequal measurement error, which are assumed to be normally distributed. The term $\delta$ explains any unknown measurement error. Any noise component that cannot be modelled is described by the term $\delta$. The probability distribution of $\delta$ is chosen to be a Gaussian distribution with finite variance $s^{2}$. Therefore, the combination of uncertainties $\epsilon_{i}+\delta$ has a Gaussian distribution with a variance equal to $\sigma_{i}^{2}+s^{2}$ (see Balan \& Lahav 2009, for more details).

The parameter estimation in the Bayesian analysis needs a choice of priors. We choose the priors following the studies by Ford \& Gregory (2007), Balan \& Lahav (2009). The mathematical form of the prior is given in Table 1 and/or 4 of Balan \& Lahav (2009). In Table 4, we provide the parameter boundaries explored in the Markov chain Monte Carlo (MCMC) Bayesian analysis. EXOFIT performs 100 chains of 10000 iterations each resulting in a final chain of 19600 sets of global-fit parameters.

We want to simultaneously model the stellar rotation and planetary signals. For that we use the ExoFIT to model two RV signals and for the rotation signal we also leave the eccentricity as a free parameter. The posterior distribution of the eccentricity parameter for the rotation signal (not shown in Fig. 13) gives a value of $0.47 \pm 0.26$. In Fig. 13 we depict the posterior distribution of the model parameters; the six fitted parameters; the semi-amplitude velocity, $K_{\text {rot }}$, and the period, $P_{\text {rot }}$, of the rotation signal; the derived mass of the planet, $m_{\mathrm{p}} \sin i$; and the RV noise given by the $s$ parameter. Most of the parameters show symmetric density profiles except for the eccentricity, $e$; the longitude of periastron, $\omega$; and the fraction $\chi$ of the orbit at which the periastron occurs. We note that the density profile of the rotation period displays a tail towards slightly lower values although the rotation period is well defined.

In Table 4 we show the final parameters and uncertainties obtained with the MCMC Bayesian analysis with the code EXOFIT.

\section{Discussion}

We detect the presence of a planet with a semi-amplitude of $2.60 \mathrm{~m} \mathrm{~s}^{-1}$, which - given the stellar mass of $0.52 M_{\odot}$ - converts to $\mathrm{m} \sin i$ of $5.36 M_{\oplus}$, orbiting with a period of $8.7 \mathrm{~d}$ around GJ 536, an M-type star of $0.52 M_{\odot}$ with a rotation period of $43.9 \mathrm{~d}$ that shows an additional activity signal compatible with an activity cycle shorter than 3 yr.

The planet is a small super-Earth with an equilibrium temperature $344 \mathrm{~K}$ for a Bond albedo $A=0.75$ and $487 \mathrm{~K}$ for $A=0$. Following Kasting et al. (1993) and Selsis et al. (2007), we perform a simple estimation of the habitable zone (HZ) of this star. The HZ would go from 0.2048 to 0.3975 AU in the narrowest case (cloud free model), and from 0.1044 to $0.5470 \mathrm{AU}$ in the broadest case (fully clouded model). This corresponds to orbital periods ranging from 46 to $126 \mathrm{~d}$ in the narrowest case, and from 17 to $204 \mathrm{~d}$ in the broadest one.

GJ $536 \mathrm{~b}$ is in the lower part of the mass vs. period diagram of known planets around M-dwarf stars (Fig. 14). The planet is too close to the star to be considered habitable. For this star the orbital periods of the habitable zone would be from $\sim 20 \mathrm{~d}$ to $\sim 40 \mathrm{~d}$.

GJ536 is a quiet early $\mathrm{M}$ dwarf, with a rotation period at the upper end of the stars of its kind (Newton et al. 2016; Suárez Mascareño et al. 2016). Its rotation induced radialvelocity signal has a semi-amplitude of $2.26 \mathrm{~m} \mathrm{~s}^{-1}$ and seems to be stable enough to allow for a clean enough periodogram and 

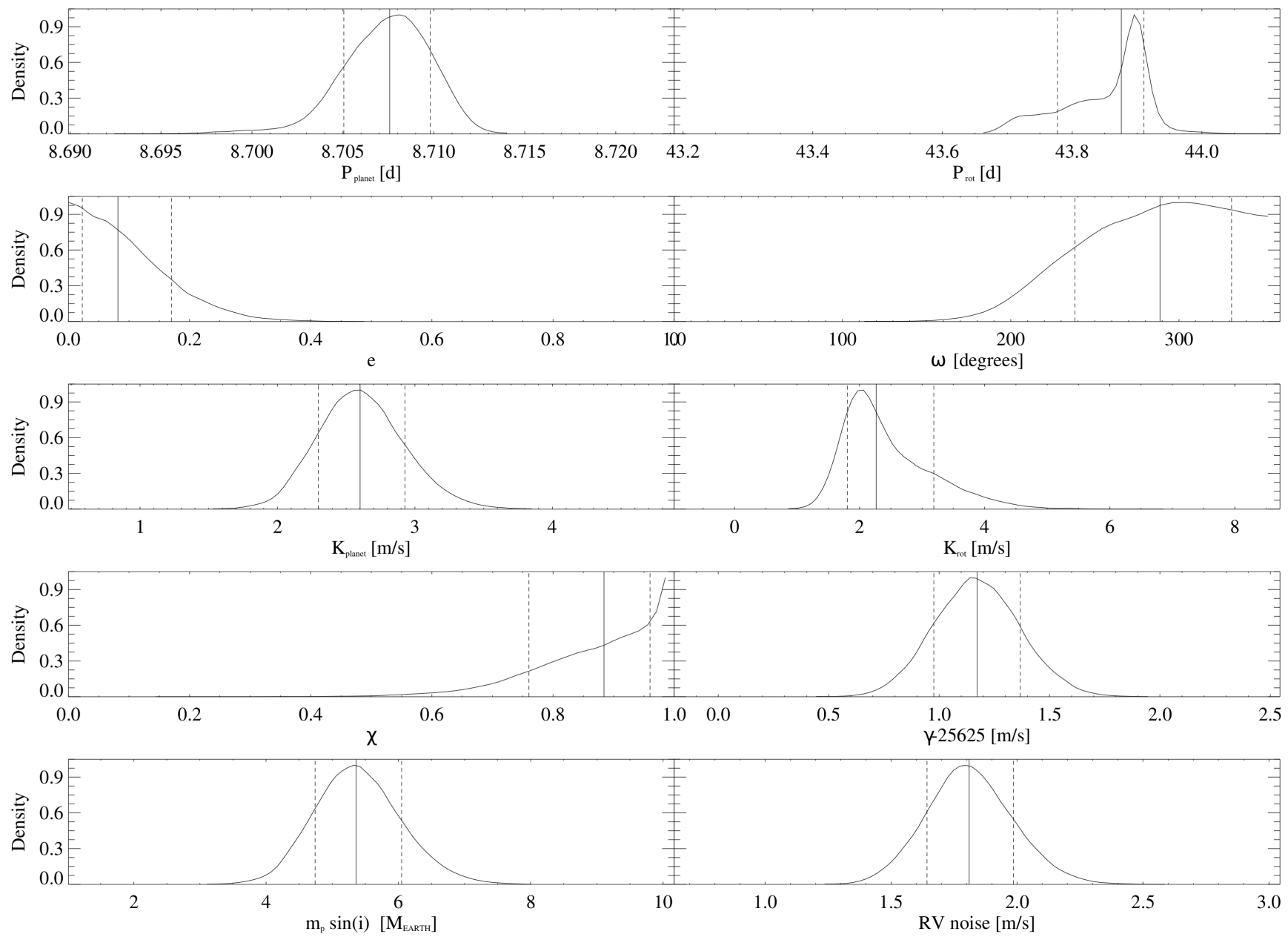

Fig. 13. Posterior distribution of model parameters including the activity signal associated with the rotation period and the orbital parameters of the planet companion of the M-dwarf star GJ 536. The vertical dashed line shows the median value of the distribution and the dotted lines the $1 \sigma$ values.

Table 4. MCMC parameters and uncertainties.

\begin{tabular}{lcccc}
\hline \hline Parameter & Value & Upper error & Lower error & Prior \\
\hline$P_{\text {planet }}[\mathrm{d}]$ & 8.7076 & +0.0022 & -0.0025 & $8.3-9.0$ \\
$\gamma\left[\mathrm{m} \mathrm{s}^{-1}-25625\right]$ & 1.17 & +0.20 & -0.20 & $-5.0-+5.0$ \\
$e$ & 0.08 & +0.09 & -0.06 & $0.0-0.99$ \\
$\omega[\mathrm{deg}]$ & 288.7 & +42.5 & -50.6 & $0.0-360.0$ \\
$\chi$ & 0.88 & +0.08 & -0.12 & $0.0-0.99$ \\
$K_{\text {planet }}\left[\mathrm{ms}^{-1}\right]$ & 2.60 & +0.33 & -0.30 & $0.0-5.0$ \\
$a[\mathrm{AU}]$ & 0.066610 & +0.000011 & -0.000013 & - \\
$m_{\mathrm{p}} \sin i\left[M_{\text {Earth }}\right]$ & 5.36 & +0.69 & -0.62 & - \\
$P_{\text {rot }}[\mathrm{d}]$ & 43.88 & +0.03 & -0.10 & $42.5-45.0$ \\
$K_{\text {rot }}\left[\mathrm{ms}^{-1}\right]$ & 2.26 & +0.92 & -0.46 & $0.0-7.0$ \\
RV noise $\left[\mathrm{ms}^{-1}\right]$ & 1.81 & +0.18 & -0.17 & $0.0-5.0$ \\
\hline
\end{tabular}

to be correctly characterized. The phase of the rotation induced signal seems to be advanced by $\sim 45^{\circ}$ with respect to the signals in the $S_{M W}$ index and FWHM time series. There is a hint of an activity cycle shorter than $3 \mathrm{yr}$, which would put it at the lower end of the stars of its kind (Suárez Mascareño et al. 2016), and whose amplitude is so small that would need further follow-up to be properly characterized. The radial-velocity signal induced by this cycle at this point is beyond our detection capabilities.

Given the rms of the residuals there is still room for the detection of more planets in this system, especially at orbital periods longer than the rotation period. Figure 14 shows the upper limits to the mass of those hypothetical companions. The stability of its rotation signals and the low amplitude of the radialvelocity signals with a magnetic origin makes this star a good candidate to search for longer period planets of moderate mass. A rough estimate of the detection limits tells us there is still room for Earth-like planets $\left(\sim 1 M_{\oplus}\right)$ at orbits smaller than 10, superEarths $\left(<10 M_{\oplus}\right)$ at orbits going from 10 to $400 \mathrm{~d}$, and even for a Neptune-mass planet $\left(<20 M_{\oplus}\right)$ at periods longer than $\sim 3 \mathrm{yr}$. Giant planets, on the other hand, are discarded except for those 


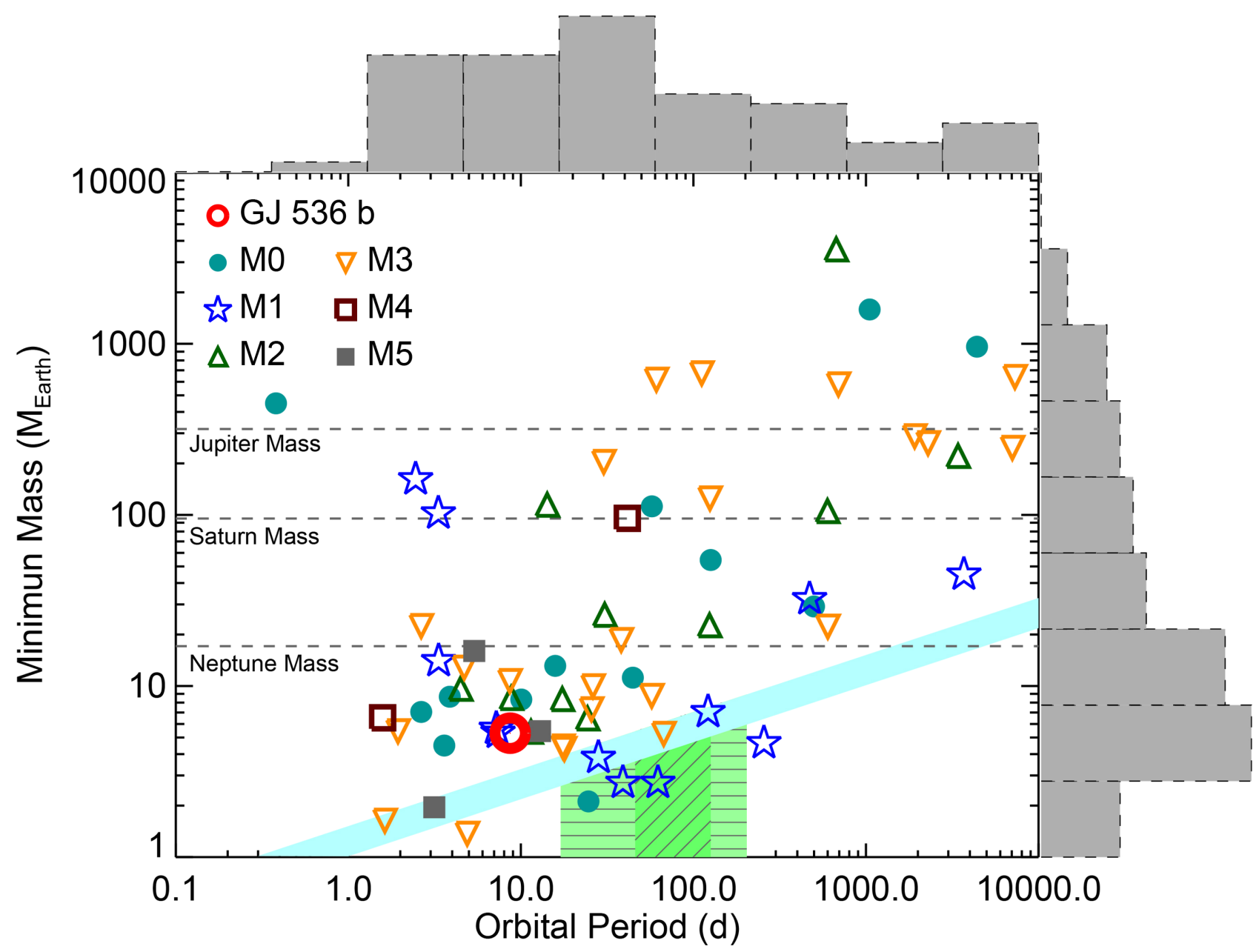

Fig. 14. Minimum mass vs. orbital period for the known planets around M-dwarf stars. The red empty dot shows the position of GJ 536 b. Horizontal dashed lines show the mass of the solar system planets for comparison. On the edges of the figure the distribution for each parameter is shown. The cyan shape shows the region where a second planet could exist around GJ 536 (upper limit of the band) and could be detectable given the typical uncertainty of the measurements (lower limit of the band). The green shapes show the habitable region around the star GJ 536. Horizontal lines show the broad scenario, while inclined lines show the narrow one.

with extremely long orbital periods. The time-span of the observations and the RMS of the residuals completely rules out the presence of any planet bigger than twice the mass of Neptune with an orbital period shorter than $\sim 20 \mathrm{yr}$.

\section{Conclusions}

We have analysed 152 high-resolution spectra and 359 photometric observations to study the planetary companions around the M-dwarf star GJ 536 and its stellar activity. We detected two significant radial-velocity signals at periods of 8.7 and $43.8 \mathrm{~d}$, respectively.

From the available photometric and spectroscopic information we conclude that the $8.7 \mathrm{~d}$ signal is caused by a $5.3 M_{\oplus}$ planet with semi-major axis of $0.067 \mathrm{AU}$ and equilibrium temperature lower than $500 \mathrm{~K}$. The short period of the planet makes it a potential transiting candidate. Detecting the transits would give a new constraining point to the mass-radius diagram.

The second radial-velocity signal of period $43.8 \mathrm{~d}$ and semiamplitude of $1.6 \mathrm{~m} \mathrm{~s}^{-1}$ is a magnetic activity induced signal related to the rotation of the star. We also found a magnetic cycle shorter than $3 \mathrm{yr}$ which would place this star among those with the shortest reported magnetic cycles.
We have studied and set limits to the presence of other planetary companions taking into account the rms of the residuals after fitting both the planet and the rotation induced signal. The system still has room for other low-mass companions, but planets more massive than Neptune are discarded except at extremely long orbital periods beyond the habitable zone of the star.

Acknowledgements. This work has been financed by the Spanish Ministry project MINECO AYA2014-56359-P. J.I.G.H. acknowledges financial support from the Spanish MINECO under the 2013 Ramón y Cajal program MINECO RYC-2013-14875. X.B., X.D., T.F., and F.M. acknowledge the support of the French Agence Nationale de la Recherche (ANR) under the programme ANR12-BS05- 0012 Exo-atmos. X.B. and A.W. acknowledge funding from the European Research Council under the ERC Grant Agreement No. 337591ExTrA. This work was supported by Fundação para a Ciência e a Tecnologia (FCT) within projects reference PTDC/FIS-AST/1526/2014 (POCI-01-0145FEDER-016886) and UID/FIS/04434/2013 (POCI-01-0145-FEDER-007672). N.C.S. acknowledges support through Investigador FCT contract of reference IF/00169/2012, and POPH/FSE (EC) by FEDER funding through the program "Programa Operacional de Factores de Competitividade - COMPETE". This work is based on data obtained via the HARPS public database at the European Southern Observatory (ESO). This research has made extensive use of the SIMBAD database, operated at CDS, Strasbourg, France, and NASA's Astrophysics Data System. We are grateful to all the observers of the following ESO projects, whose data we are using: 072.C-0488, 085.C-0019, 183.C-0972, and 191.C-087. 


\section{References}

Affer, L., Micela, G., Damasso, M., et al. 2016, A\&A, 593, A117 Anglada-Escudé, G., Amado, P. J., Barnes, J., et al. 2016, Nature, 536, 437 Astudillo-Defru, N., Bonfils, X., Delfosse, X., et al. 2015, A\&A, 575, A119 Balan, S. T., \& Lahav, O. 2009, MNRAS, 394, 1936

Baliunas, S. L., Donahue, R. A., Soon, W. H., et al. 1995, ApJ, 438, 269

Baranne, A., Queloz, D., Mayor, M., et al. 1996, A\&AS, 119, 373

Berdyugina, S. V., \& Järvinen, S. P. 2005, Astron. Nachr., 326, 283

Berta-Thompson, Z. K., Irwin, J., Charbonneau, D., et al. 2015a, Nature, 527, 204

Berta-Thompson, Z. K., Irwin, J., Charbonneau, D., et al. 2015b, Nature, 527, 204

Bonfils, X., Mayor, M., Delfosse, X., et al. 2007, A\&A, 474, 293

Bonfils, X., Delfosse, X., Udry, S., et al. 2013, A\&A, 549, A109

Cosentino, R., Lovis, C., Pepe, F., et al. 2012, in Ground-based and Airborne Instrumentation for Astronomy IV, SPIE, 8446, 84461

Cumming, A. 2004, MNRAS, 354, 1165

Delfosse, X., Forveille, T., Perrier, C., \& Mayor, M. 1998, A\&A, 331, 581

Delfosse, X., Bonfils, X., Forveille, T., et al. 2013, A\&A, 553, A8

Dressing, C. D., \& Charbonneau, D. 2013, ApJ, 767, 95

Dressing, C. D., Charbonneau, D., \& Newton, E. R. 2015, in AAS/Division for Extreme Solar Systems Abstracts, 3, 501.03

Endl, M., Kürster, M., Els, S., Hatzes, A. P., \& Cochran, W. D. 2001, A\&A, 374, 675

Ford, E. B. 2005, AJ, 129, 1706

Ford, E. B., \& Gregory, P. C. 2007, in Statistical Challenges in Modern Astronomy IV, eds. G. J. Babu, \& E. D. Feigelson, ASP Conf. Ser., 371, 189 Gaidos, E. 2013, ApJ, 770, 90

Gomes da Silva, J., Santos, N. C., Bonfils, X., et al. 2011, A\&A, 534, A30

Gregory, P. C. 2005, ApJ, 631, 1198

Horne, J. H., \& Baliunas, S. L. 1986, ApJ, 302, 757

Howard, A. W., Johnson, J. A., Marcy, G. W., et al. 2009, ApJ, 696, 75

Howard, A. W., Marcy, G. W., Bryson, S. T., et al. 2012, ApJS, 201, 15

Howard, A. W., Marcy, G. W., Fischer, D. A., et al. 2014, ApJ, 794, 51

Irwin, J., Berta-Thompson, Z. K., Charbonneau, D., Dittmann, J., \& Newton, E. R. 2015, in AAS Meet. Abstr., 225, 258.01

Kasting, J. F., Whitmire, D. P., \& Reynolds, R. T. 1993, Icarus, 101, 108

Koen, C., Kilkenny, D., van Wyk, F., \& Marang, F. 2010, MNRAS, 403, 1949

Kopparapu, R. K. 2013, ApJ, 767, L8

Lovis, C., Dumusque, X., Santos, N. C., et al. 2011, unpublished [arXiv: 1107.5325]

Maldonado, J., Affer, L., Micela, G., et al. 2015, A\&A, 577, A132
Marcy, G. W., Butler, R. P., Vogt, S. S., Fischer, D., \& Lissauer, J. J. 1998, ApJ, 505, L147

Markwardt, C. B. 2009, in Astronomical Data Analysis Software and Systems XVIII, eds. D. A. Bohlender, D. Durand, \& P. Dowler, ASP Conf. Ser., 411, 251

Mayor, M., Pepe, F., Queloz, D., et al. 2003, The Messenger, 114, 20

Mayor, M., Bonfils, X., Forveille, T., et al. 2009, A\&A, 507, 487

Mayor, M., Marmier, M., Lovis, C., et al. 2011, A\&A, submitted [arXiv: 1109.2497]

Montet, B. T., Crepp, J. R., Johnson, J. A., Howard, A. W., \& Marcy, G. W. 2014, ApJ, 781, 28

Newton, E. R., Irwin, J., Charbonneau, D., Berta-Thompson, Z. K., \& Dittmann, J. A. 2016, ApJ, 821, L19

Noyes, R. W., Hartmann, L. W., Baliunas, S. L., Duncan, D. K., \& Vaughan, A. H. 1984, ApJ, 279, 763

Pepe, F., Mayor, M., Delabre, B., et al. 2000, in Optical and IR Telescope Instrumentation and Detectors, eds. M. Iye, \& A. F. Moorwood, SPIE, 4008, 582

Pojmanski, G. 1997, Acta Astron., 47, 467

Queloz, D., Henry, G. W., Sivan, J. P., et al. 2001, A\&A, 379, 279

Rajpaul, V., Aigrain, S., \& Roberts, S. 2016, MNRAS, 456, L6

Reiners, A. 2009, A\&A, 498, 853

Robertson, P., Mahadevan, S., Endl, M., \& Roy, A. 2014, Science, 345, 440

Robertson, P., Endl, M., Henry, G. W., et al. 2015, ApJ, 801, 79

Saar, S. H., \& Donahue, R. A. 1997, ApJ, 485, 319

Santos, N. C., Mortier, A., Faria, J. P., et al. 2014, A\&A, 566, A35

Selsis, F., Kasting, J. F., Levrard, B., et al. 2007, A\&A, 476, 1373

Suárez Mascareño, A., Rebolo, R., González Hernández, J. I., \& Esposito, M. 2015, MNRAS, 452, 2745

Suárez Mascareño, A., Rebolo, R., \& González Hernández, J. I. 2016, A\&A, 595, A12

Udry, S., Bonfils, X., Delfosse, X., et al. 2007, A\&A, 469, L43

van Leeuwen, F. 2007, A\&A, 474, 653

Waldmeier, M. 1961, The sunspot-activity in the years 1610-1960 (Zurich: Schulthess)

Wildi, F., Pepe, F., Chazelas, B., Lo Curto, G., \& Lovis, C. 2010, in Groundbased and Airborne Instrumentation for Astronomy III, SPIE, 7735, 77354

Wright, J., \& Howard, A. 2012, Astrophysics Source Code Library, [record ascl: 1210.031]

Wright, D. J., Wittenmyer, R. A., Tinney, C. G., Bentley, J. S., \& Zhao, J. 2016, ApJ, 817, L20

Zechmeister, M., \& Kürster, M. 2009, A\&A, 496, 577

Zechmeister, M., Kürster, M., \& Endl, M. 2009, A\&A, 505, 859 